\title{
Considering Inventory Distributions in a Stochastic Periodic Inventory Routing System
}

\section{Ehsan Yadollahi, a) and El-Houssaine Aghezzaf ${ }^{2, \text { b) }}$}

\author{
${ }^{1,2}$ Department of Industrial Systems Engineering and product design, Faculty of Engineering and Architecture, \\ Ghent University, Gent, Belgium
}

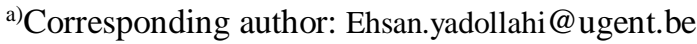

b) ElHoussaine.Aghezzaf@ugent.be

\begin{abstract}
Dealing with the stochasticity of parameters is one of the critical issues in business and industry nowadays. Supply chain planners have difficulties in forecasting stochastic parameters of a distribution system. Demand rates of customers during their lead time are one of these parameters. In addition, holding a huge level of inventory at the retailers is costly and inefficient. To cover the uncertainty of forecasting demand rates, researchers have proposed the usage of safety stock to avoid stock-out. However, finding the precise level of safety stock depends on forecasting the statistical distribution of demand rates and their variations in different settings among the planning horizon. In this paper the demand rate distributions and its parameters are taken into account for each time period in a stochastic periodic IRP. An analysis of the achieved statistical distribution of the inventory and safety stock level is provided to measure the effects of input parameters on the output indicators. Different values for coefficient of variation are applied to the customers' demand rate in the optimization model. The outcome of the deterministic equivalent model of SPIRP is simulated in form of an illustrative case.
\end{abstract}

Keywords: Inventory Routing Problem (IRP), Simulation, Statistical Distribution, Safety Stock

\section{INTRODUCTION AND LITERATURE}

Stochastic demand rates are considered in IRP to involve the effects of demand's uncertainty in optimizing process. In this sense, the demand rates of customers are known by the supplier and are following probabilistic distributions. In case the demand rate for a certain time period is higher than delivered and/or in-hand products, stock-out will occur. Therefore there is a necessity to store a small amount of products as safety stock at the customer. In the other hand, reducing the cost forces supply chain planners to minimize the level of safety stock which results in higher probability of stock-out occurrence, that brings lower level of service for both system and customers.

Researchers have already developed numerous models based on SIRP to find the balance between amount of safety stock and service level (Dror and Ball, 1987, Abdul Rahim et al., 2014, Aghezzaf, 2007, Campbell et al., 1998, Kleywegt et al., 2004). Taking into account infinite time horizon, (Jaillet et al., 2002) developed a model on 
SIRP based on the framework of (Bard et al., 1998) to minimize the total expected annual delivery costs. Simplifying the SIRP model caused (Campbell et al., 1998) to develop a dynamic programming model in which only transportation and stock-out costs are taken into consideration. In their model, inventory holding costs are not incurred. (Kleywegt et al., 2004) determined optimal policies for SIRP in which a single product is distributed from a single vendor to multiple customers. They involved inventory costs as well as transportation and shortage costs over an infinite horizon to have a comprehensive assessment. Markov decision process is applied to formulate SIRP in their research. (Adelman, 2004) dealt with the problem in a different way. He presents a linear program that takes into account inventory dynamics and economics in allocating transportation costs in stochastic inventory routing problem. In compare to (Kleywegt et al., 2004), the constraints in the number of customers in a tour is removed in (Adelman, 2004) research that results in a more general model.

Forecasting is the process of predicting the future based on past and present data and analysis of trends. A commonplace example might be estimation of some variable of interest at some specified future date. Evaluating the trends and parameters in a long term planning horizon helps the supply chain planners to estimate the future behavior of the system. Predicting (close to) real demand rates for the planning horizon would allow the decision maker to decrease the level of safety stock in long term and save extra costs dependent on inventory level and transportation.

In this paper to have a better understanding of the behaviour of stock-out, safety stock and inventory level in a stochastic periodic inventory routing problem, we define some indicators to measure their changes and trends during the planning horizon. Several customers in a distribution system are taken into account with different demand rates and coefficient of variations. According to the defined setting for replenishment system, the outcome of the model will be evaluated and analysed.

The rest of the paper is organized as follows. In section 2 the SPIRP model is presented. To examine the models and evaluate the results, in section 3 an illustrative examples is taken into consideration together with the results and discussions which also includes different sorts of evaluations and comparisons among the achieved results.

\section{SPIRP MODEL}

In this paper the studied distribution system consists of a single depot and a set of geographically scattered customers. The customers are indexed by $\mathrm{j}, \mathrm{j} \in\{1,2, \ldots, \mathrm{n}\}$ (the number of customers) and the depot is indexed by $\mathrm{r}$ . Each customer $\mathrm{j}$ has a stochastic demand rate of $\mathrm{E}\left(\mathrm{d}_{\mathrm{jt}}\right)$ per unit of time that is Normally distributed $\left(\mathrm{d}_{\mathrm{jt}} \sim\right.$ $\left.\mathrm{N}\left(\mathrm{E}\left(\mathrm{d}_{\mathrm{jt}}\right), \sigma_{\mathrm{j}}\right)\right)$ with standard deviation of $\sigma_{\mathrm{j}}$. For the deliveries, one (several) vehicle(s) with a capacity of $\kappa$ ton is available. Supplier and customers are agreed to have a service level based on a fill rate of SL percent.

Each customer is replenished every $\mathrm{T}$ days. Inventory control at this customer can be considered as a periodicreview, order-up-to-level system with backlogging. The review period is given by the cycle time of $\mathrm{T}$ days, while the replenishment lead time is assumed to be negligible, because the delivery quantity is decided just before the vehicle is dispatched. Let $H=\{1,2, \ldots, T\}$ be the planning horizon set of consecutive periods indexed by $t$, and $\mathrm{H}^{+}=\mathrm{HU}\{0\}$ that shows planning horizon include $\mathrm{t}=0$. Let $\tau_{\mathrm{t}}$ be the size in time units of each period $t$, for example eight working hours per day. Let $S$ be the set of retailers indexed by $i$ and $j$; and $S^{+}=S \cup\{r\}$. A fleet of vehicles $\mathrm{V}, \mathrm{v} \in\{1,2, \ldots, \mathrm{n}\}$ is occupied to serve the retailers.

Additional parameters of the model are as follow:

$\phi_{\mathrm{jt}}$ : the fixed handling cost (in euros) per delivery at location $\mathrm{j} \in \mathrm{S}^{+}$(retailers and depot) in period $\mathrm{t} \in \mathrm{H}$.

$\eta_{\mathrm{jt}}$ : the per unit per period holding cost of the product at location $\mathrm{j} \in \mathrm{S}^{+}$(in euros per ton per period);

$\Psi_{\mathrm{vt}}$ : the fixed operating cost of vehicle $\mathrm{v} \in \mathrm{V}$ (in euros per vehicle) in period $\mathrm{t} \in \mathrm{H}$;

$\delta_{\mathrm{v}}$ : travel cost of vehicle $\mathrm{v} \in \mathrm{V}$ (in euros per $\mathrm{km}$ );

$v_{\mathrm{v}}$ : average speed of vehicle $\mathrm{v} \in \mathrm{V}$ (in km per hour);

$\theta_{\mathrm{ij}}$ : duration of a trip from retailer $\mathrm{i} \in \mathrm{S}^{+}$to retailer $\mathrm{j} \in \mathrm{S}^{+}$(in hour);

The variables of the model are defined as follows:

$\mathrm{Q}_{\mathrm{vijt}}$ : the quantity of product remaining in vehicle $\mathrm{v} \in \mathrm{V}$ when it travels directly to location $\mathrm{j} \in \mathrm{S}^{+}$from location $i \in S^{+}$in period $t \in H$. This quantity equals zero when the trip $(i, j)$ is not on any tour of the route travelled by vehicle $\mathrm{v} \in \mathrm{V}$ in period $\mathrm{t}$;

$\mathrm{q}_{\mathrm{jt}}$ : the quantity delivered to location $\mathrm{j} \in \mathrm{S}^{+}$in period $\mathrm{t} \in \mathrm{H}$;

$I_{j t}$ : the inventory level at location (retailers and warehouse) $j \in \mathrm{S}^{+}$by the end of period $t \in \mathrm{H}^{+}$; 
$\mathrm{x}_{\mathrm{vijt}}$ : a binary variable set to 1 if location $\mathrm{j} \in \mathrm{S}^{+}$is visited immediately after location $\mathrm{i} \in \mathrm{S}^{+}$by vehicle $\mathrm{v} \in \mathrm{V}$ in period $t \in \mathrm{H}$, and 0 otherwise;

$\mathrm{y}_{\mathrm{vt}}$ : a binary variable set to 1 if vehicle $\mathrm{v} \in \mathrm{V}$ is being used in period $\mathrm{t}$, and 0 otherwise;

$\mathrm{I}_{\mathrm{j} 0}$ : the initial inventory levels at each retailer $\mathrm{j} \in \mathrm{S}$ in period zero;

$\mathrm{I}_{\mathrm{jT}}$ : Inventory levels at retailer $\mathrm{j} \in \mathrm{S}$ in the last period;

To minimize:

$$
C V=\sum_{t \in H} \sum_{v \in V}\left[\psi_{v t} y_{v t}+\sum_{i \in S^{+}} \sum_{j \in S^{+}}\left(\delta_{v} v_{v} \theta_{i j}+\phi_{j t}\right) x_{v i j t}\right]+\sum_{t \in H^{+}} \sum_{j \in S^{+}} \eta_{j t} I_{j t}
$$

Subject to:

$$
\begin{gathered}
\sum_{v \in V} \sum_{i \in S^{+}} x_{v i j t} \leq 1, \quad \forall j \in S, t \in H \\
\sum_{i \in S^{+}} x_{v i j t}-\sum_{k \in S^{+}} x_{v j k t}=0, \quad \forall j \in S^{+}, t \in H, \quad v \in V \\
\sum_{i \in S^{+}} \sum_{j \in S^{+}} \theta_{i j} x_{v i j t} \leq \tau_{t}, \quad \forall t \in H, v \in V \\
\sum_{v \in V} \sum_{i \in S^{+}} Q_{v i j t}-\sum_{v \in V} \sum_{k \in S^{+}} Q_{v j k t}=q_{j t}, \quad \forall j \in S, \quad t \in H \\
Q_{v i j t} \leq \kappa x_{v i j t}, \quad \forall i \in S^{+}, j \in S^{+}, t \in H, v \in V \\
I_{j 0}+\sum_{s=1}^{t} q_{j s}=\sum_{s=1}^{t} E\left(d_{j s}\right)+S S_{j t}+I_{j t}, \quad \forall j \in S, t \in H^{+} \\
x_{v i j t} \leq y_{v t}, \quad \forall j \in S^{+}, t \in H, v \in V \\
x_{j 0} \leq I_{j T}, \quad \forall j \in S, t \in H^{+} \\
x_{v i j t} \geq 0, q_{j t} \geq 0, \quad \forall j \in S^{+}, t \in H, v \in V
\end{gathered}
$$

The objective function (1) shows the variables and fix indicators which have been used in the model to minimize the level of costs in this replenishment system. It includes four cost components, namely, total fixed operating cost of using the vehicle(s), total transportation cost, total delivery handling cost and total inventory holding cost at the end of each period. 
Constraints (2) make sure that each customer is visited not more than once in each period. Constraints (3) focus on the vehicle to move to the next customer/depot after serving it. Each tour must be less than or equal to the time considered for one period and it is considered by constraints (4) to prevent tours that take more than one period. The quantities to be delivered for each customer are determined by constraints (5) which also avoid sub-tour happening. Constraints (6) are the limitations for vehicle capacity. Constraints (7) determine the level of inventory at the beginning of model (period zero) and end of each period. In addition they assure the level of initial inventory, safety stock, and delivery quantity in each period should be equal to demand rates and remaining inventory at the end of that period. This constraint is stochastic and complicates the problem. Constraints (8) insure the level of inventory at the end of last period is equal or larger than initial inventory. And finally constraints (9) specify a vehicle cannot be occupied to serve retailers unless it is chosen.

This model is looking for the optimum level of delivery quantity based on the standard deviation of expected demand rates in a certain level of service in planning horizon. According to the constraints (7) the delivery quantity for each customer is calculated based on both stochastic demand rates, level of safety stock $\left(S S_{j t}\right)$ during each period, and last period's inventory level. Safety stock (equation 10) is applied to the model to prevent stock-out. The amount of safety stock and its allocation mechanism during a long term planning horizon is explained in below.

As we explained before we use safety stock to diminish the fluctuations of demand rates. The equation to calculate safety stock is presented as follow:

$$
S S_{j t}=z_{\alpha} * \sqrt{\sum_{s=1}^{t} \sigma_{j s}^{2}}
$$

As it is shown in equation 10 , safety stock is a function of service level parameter $\left(z_{\alpha}\right)$, number of time periods $(t)$, and standard deviation $\left(\sigma_{j t}\right)$ for each customer. $z_{\alpha}$ is the safety factor for service level $(S L)$ gained by the level of $\alpha$. In case of the normal distribution, $z_{\alpha}$ is the probability of $(1-\alpha / 2) . \alpha$ is the percentage of expected stock-out for each customer during the whole planning horizon.

\subsection{Indicators to Measure the Models' Performance}

\section{Excess inventory $\left(I_{j}^{e}\right)$ at the end of the planning horizon}

We measure $I_{j}^{e}$ at the end of the planning horizon to check whether the model has behaved in order to satisfy constraints (8). In the mathematical model, the stock at the end of the planning horizon should be larger or equal than the initial stock. This indicator measures the difference of inventory between all proposed models for customer $j$ after simulating the results of optimization model. The average level of the remaining inventory shows how (in)efficient it is to allocate safety stock. Equation (11) measures the value for $I_{j}^{e}$ as follow:

$$
I_{j}^{e}=I_{j T}-I_{j 0}
$$

\section{Excess stock $\left(S S_{j t}^{e}\right)$ at the end of each period}

$S S_{j t}^{e}$ measures the level of stock remaining at the customer $j$ at the end of period $t$. This indicator specifies whether safety stock is allocated in each period and shows fluctuation coverage of safety stock. Below you see equation (12) to calculate $S S_{j t}^{e}$ :

$$
\left.S S_{j t}^{e}=I_{j 0}+\sum_{s=1}^{t} q_{j s}-I_{j t}-\sum_{s=1}^{t}\left(d_{j s}\right) \tau_{s}\right)
$$

In this equation, basically, if the demand rates are constant, the outcome for $S S_{j t}^{e}$ would be zero. But because demand rates are assumed to be Normally distributed, this value would be either positive or negative. If the value for $S S_{j t}^{e}$ is positive, it means there are enough products at customer $j$ during period $t$ to cover extra quantity in demand 
due to the uncertainties. On the other hand, when $S S_{j t}^{e}$ is negative, it means there is no extra safety stock to be transferred to the next period. In this condition, stock-out may happens if there is not enough stock left from previous period. Therefore finding the balance between service level and the level of $S S_{j t}^{e}$ would be necessary to prevent high inventory costs or backlog/lost sales issues.

\section{ILLUSTRATIVE CASE}

The case shows a distribution centre with one central depot and 5 scattered customers around it. Defined indicators will be measured after 200 replications of simulation. Different customers have different coefficient of variations. They have normally distributed demand rates and the planning is done for 24 time periods. Three homogeneous vehicles with 80 tons capacity do the replenishments.

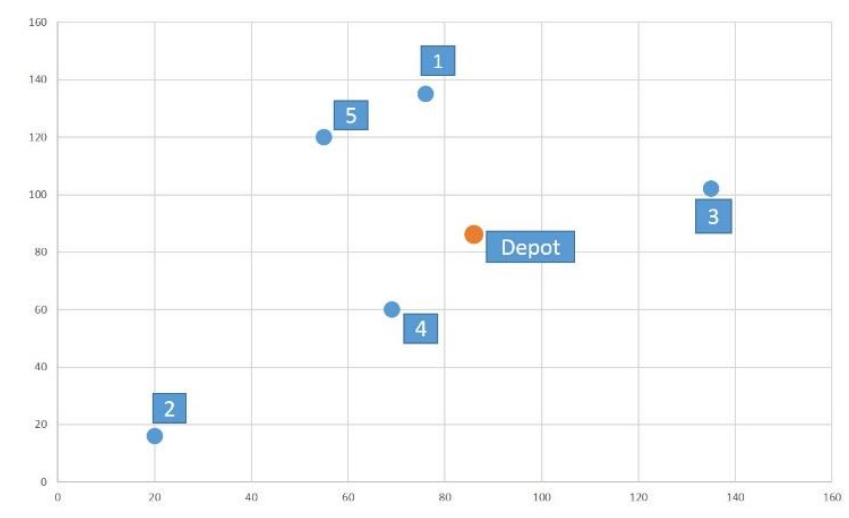

Figure 1: Chematic view of the distribution center

In figure 1 you see the schematic view of the distribution system which is consisting of one warehouse (depot) and 5 customers around it. The average, standard deviations and coefficient of variations are presented in table 1. Due to the uncertainty in demand rates, there is possibility of stock-out and which is considered as fully backlogged. The distances between the customers are available in table 2. The planning horizon is for 24 periods. The rest of the parameters of this example are provided in table 3.

Table 1: distances between customers and depot

\begin{tabular}{|c|c|c|c|c|c|c|}
\hline & Depot & \multicolumn{1}{l|}{$\mathrm{c1}$} & $\mathrm{c} 2$ & $\mathrm{c} 3$ & $\mathrm{c} 4$ & $\mathrm{c5}$ \\
\hline Depot & 0 & 0,5001 & 0,9621 & 0,5155 & 0,3106 & 0,4601 \\
\hline $\mathrm{c1}$ & 0,5001 & 0 & 13.152 & 0,676 & 0,7533 & 0,2581 \\
\hline $\mathrm{c} 2$ & 0,9621 & 13.152 & 0 & 1.436 & 0,6586 & 10.973 \\
\hline $\mathrm{c3}$ & 0,5155 & 0,676 & 1.436 & 0 & 0,7823 & 0,82 \\
\hline $\mathrm{c} 4$ & 0,3106 & 0,7533 & 0,6586 & 0,7823 & 0 & 0,6161 \\
\hline $\mathrm{c5}$ & 0,4601 & 0,2581 & 10.973 & 0,82 & 0,6161 & 0 \\
\hline
\end{tabular}

Table 2: input level of average demand and standard deviation

\begin{tabular}{|c|c|c|c|}
\hline $\begin{array}{l}\text { Customers } \\
\text { number }\end{array}$ & $\begin{array}{l}\text { Average } \\
\text { demand per } \\
\text { period }\end{array}$ & $\begin{array}{l}\text { Standard } \\
\text { deviation } \\
(\text { Std })\end{array}$ & $\begin{array}{l}\text { Coefficient } \\
\text { of variation } \\
(\mathrm{CV})\end{array}$ \\
\hline 1 & 13 & 5 & 0.3864 \\
\hline 2 & 12 & 3 & 0.25 \\
\hline 3 & 13.5 & 0.5 & 0.037 \\
\hline 4 & 17 & 4 & 0.2352 \\
\hline
\end{tabular}




\begin{tabular}{l|l|l|}
\hline 16 & 3.5 & 0.2187 \\
\hline
\end{tabular}

Table 3: Extra information for the illustrative case

\begin{tabular}{|l|l|}
\hline Delivery costs & 25 \\
\hline $\begin{array}{l}\text { Inventory holding costs per unit } \\
\text { per period }\end{array}$ & 0.1 \\
\hline $\begin{array}{l}\text { Travel costs for vehicle in Euro per } \\
\text { KM }\end{array}$ & 1 \\
\hline Fix operating cost of vehicle & 50 \\
\hline Average speed of vehicle & 50 \\
\hline
\end{tabular}

Within each period, the vehicle visits one or more than one customers to deliver the demanded products. Loading and unloading times are not taken into account. The objective function of this model is provided to minimize the inventory and transportation costs while all the customers are supposed to receive the demanded materials on time and avoiding backlog occurrence based on a certain service level.

After optimizing the mathematical model, the solution of SPIRP on the illustrative case is applied and simulated to evaluate the solution based on randomly generated numbers. We run the simulation model for 200 replications to reduce the chance of inaccuracy.

Results and discussion:

The optimization model gives us the delivery quantity, and expected level of inventory during each time period. We use these quantity to simulate the routing and distribution system for this considered case. The simulation model generates random numbers for demand rates and illustrates the system's behaviour in different situation. 200 replications are taken into account in this simulation. The results are displayed in diagrams according to the defined indicators. Here we explain whether the model behaves.

Figure Error! Reference source not found. shows the distributions of excess inventory at the end of the planning horizon for each customer. The diagram displays the difference between the customers based on their level of CV. The higher value of CV they have, the larger the variety of excess inventory is for them. In other words, CV has a direct correlation with the range of remained inventory at the end of planning horizon. Customer number 3 has the lowest CV and as we see in figure Error! Reference source not found. and table ... (CV, average, and standard deviations for all the customers), the average and standard deviations are the lowest compare to other customers.

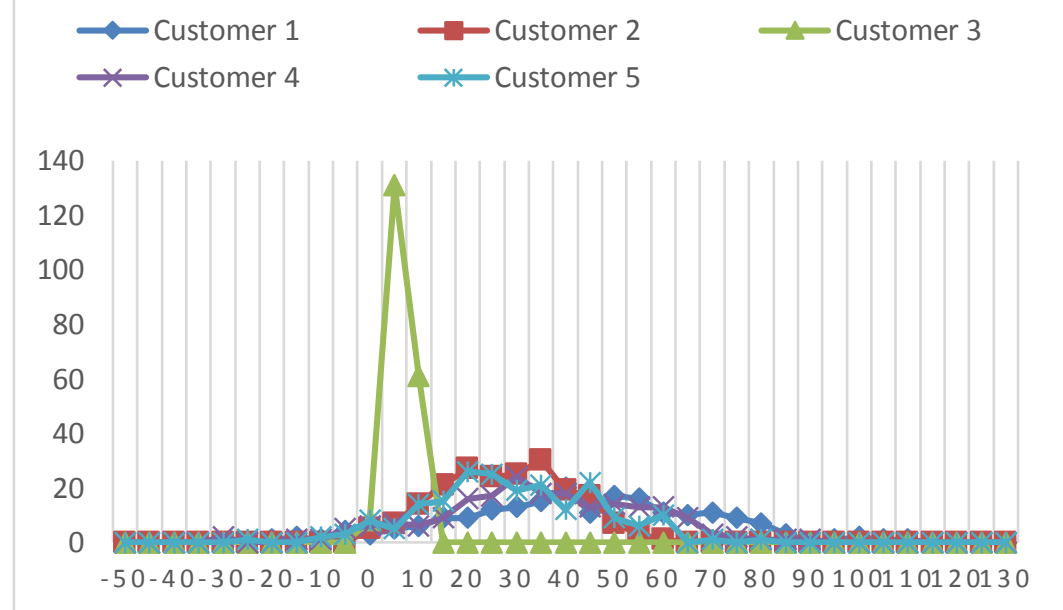

Figure 2: Excess inventory at the end for all the customers

Table 4: Achieved numbers for $\mathrm{I}_{\mathrm{j}}^{\mathrm{e}}$

\begin{tabular}{|l|l|l|l|}
\hline Customers & Average & Std & CV \\
\hline 1 & 40,6475 & 24,749 & 0,6088 \\
\hline 2 & 25,3167 & 12,795 & 0,5054 \\
\hline 3 & 3,89734 & 2,1997 & 0,5644 \\
\hline
\end{tabular}




\begin{tabular}{|l|l|l|l|}
\hline 4 & 32,2206 & 21,058 & 0,6535 \\
\hline 5 & 26,3214 & 17,316 & 0,6578 \\
\hline
\end{tabular}

Average, standard deviation and coefficient of variations are calculated for $\mathrm{SS}_{\mathrm{jt}}^{\mathrm{e}}$ for all the customers in the model. But to check whether the level of CV would affect the results, we choose only two customers 1 and 3 which have highest and lowest level of CV (table ...).

\section{$S S_{j t}^{e}$ Distribution}

The $\mathrm{SS}_{\mathrm{jt}}^{\mathrm{e}}$ or extra safety stock is an indicator that shows the difference between the expected and actual level of stock at the customer at the end of each period. This value has various behaviour among the time periods according to the coefficient of variations of customers' demand. To have analyse the trends, we consider the distributions of $\mathrm{SS}_{\mathrm{jt}}^{\mathrm{e}}$ in some periods for customers 1 and 3. Figures 3 and 4 show respectively the achieved distributions of these two customers for periods $1,6,12,18$, and 24 . Figure 3 indicates the normally distributed values of $S_{j \mathrm{jt}}^{\mathrm{e}}$ during period 1. Also the standard deviation is quite low compare to other periods. By increasing the number of periods, the average value and standard deviation increase as well. Therefore in period 24, we have the highest average and standard deviation for $\mathrm{SS}_{\mathrm{jt}}^{\mathrm{e}}$ in customer 1.

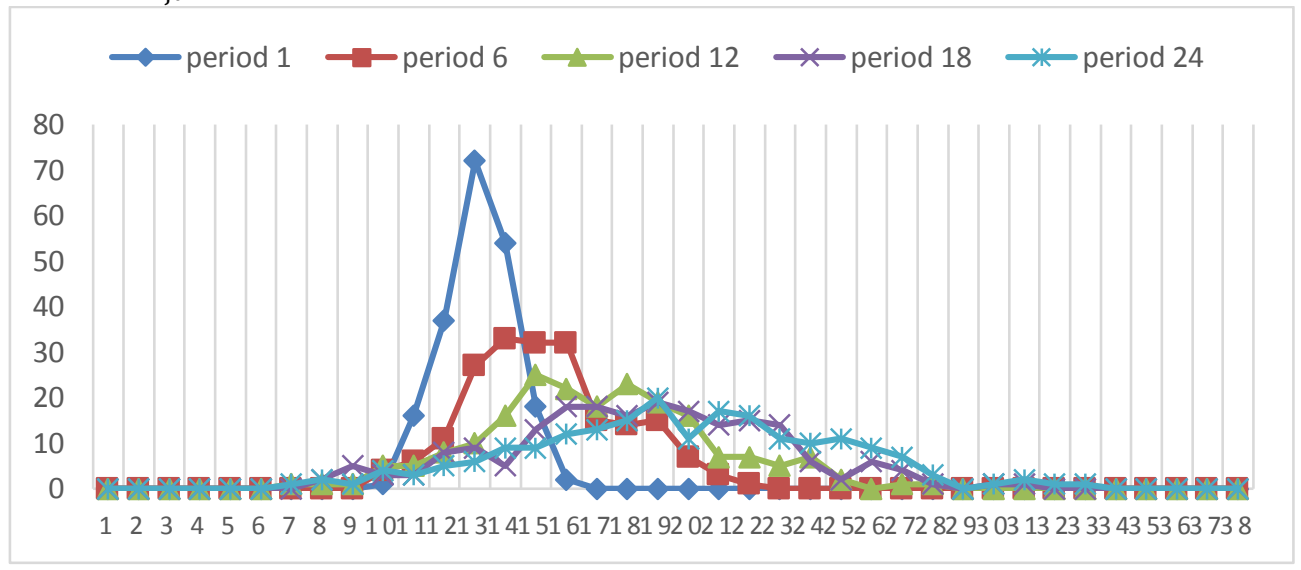

Figure 3: Customer 1 with $\mathrm{CV}=0.3846$

On the other hand, the level of $\mathrm{SS}_{\mathrm{jt}}^{\mathrm{e}}$ shows different behaviour in customer 3. Figure 4 displays the extra stock distributions among several time periods. Due to the small coefficient of variation, the achieved distributions in this customer are very close to each other from average and standard deviation perspectives. There are some small differences between the lines that shows the growing trend in average value among the planning horizon. In addition, the volume of $\mathrm{SS}_{\mathrm{jt}}^{\mathrm{e}}$ is noticeably low compare to customer 1.

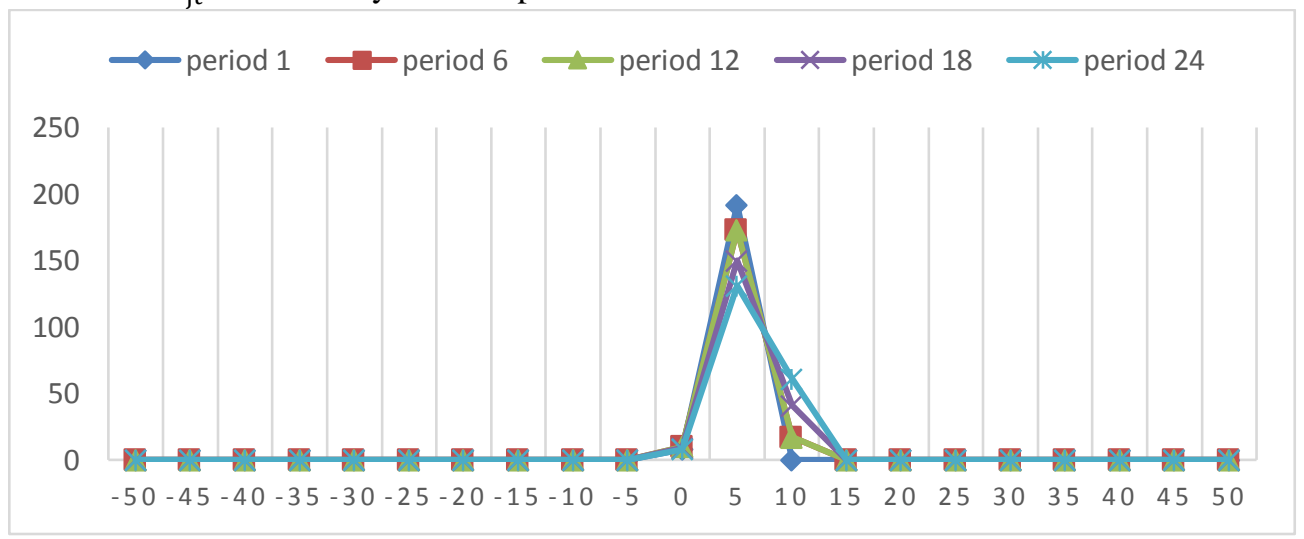

Figure 4: Customer $3 \mathrm{CV}=0.037$ 


\section{CONCLUSION}

In this paper we are considering stochastic periodic inventory routing problem in a distribution system, when demand rates are normally distributed, but with different coefficient of variance between the customers in the system. The idea is to evaluate the customers based on the defined indicators to measure their changes. The presented model is a deterministic equivalent of stochastic IRP which minimizes the level of inventory at the customers in a certain level of service, and selects the routing system for products replenishment. The outcome of model is simulated and evaluated for 200 replications on an illustrative example of distribution system. The results of the simulation have shown high correlation between CV and level of inventory at the customers. Also by increasing $\mathrm{CV}$, instability of $\mathrm{SS}_{\mathrm{jt}}^{\mathrm{e}}$ increases that causes uncertainty in forecasting excess inventory and higher volume of stock-out during the planning horizon. Moreover, the level and variance of $\mathrm{SS}_{\mathrm{jt}}^{\mathrm{e}}$ grows among the planning horizon. In other words, by increasing the number of periods, the average and standard deviation of $\mathrm{SS}_{\mathrm{jt}}^{\mathrm{e}}$ increases. The level of actual CV (measured after simulation) is always the same among the time periods, and even between the customers with different expected CV. Future research will be more about the possibilities to reduce the level of stock-out and inventory while service level is covered.

\section{REFERENCES}

Dror, M. and M. Ball, Inventory/routing: Reduction from an annual to a short-period problem. Naval Research Logistics (NRL), 1987. 34(6): p. 891-905

Abdul Rahim, M.K.I., et al., Modelling and solving the multiperiod inventory-routing problem with stochastic stationary demand rates. International Journal of Production Research, 2014. 52(14): p. 4351-4363.

Aghezzaf, E.H., Robust distribution planning for supplier-managed inventory agreements when demand rates and travel times are stationary. J Oper Res Soc, 2007. 59(8): p. 1055-1065.

Campbell, A., et al., The Inventory Routing Problem, in Fleet Management and Logistics, T. Crainic and G. Laporte, Editors. 1998, Springer US. p. 95-113.

Kleywegt, A.J., V.S. Nori, and M.W.P. Savelsbergh, Dynamic Programming Approximations for a Stochastic Inventory Routing Problem. Transportation Science, 2004. 38(1): p. 42-70.

Jaillet, P., et al., Delivery cost approximations for inventory routing problems in a rolling horizon framework. Transportation Science, 2002. 36(3): p. 292-300.

Bard, J.F., et al., A Decomposition Approach to the Inventory Routing Problem with Satellite Facilities. Transportation Science, 1998. 32(2): p. 189-203.

Adelman, D., A Price-Directed Approach to Stochastic Inventory/Routing. Operations Research, 2004. 52(4): p. 499-514. 